\title{
Singlet oxygen addition to cyclo-1,3-hexadienes from natural sources and from organocatalytic enal dimerization
}

\author{
Axel G. Griesbeck,* Alan de Kiff, Jörg M. Neudörfl, and Sarah Sillner \\ Department of Chemistry, Greinstr. 4, D-50939 Köln / Cologne, Germany \\ E-mail: griesbeck@uni-koeln.de
}
Dedicated to Michael Orfanopoulous on the occasion of his retirement and his $67^{\text {th }}$ birthday

DOI: http://dx.doi.org/10.3998/ark.5550190.p008.994

\begin{abstract}
The photoxygenation reactions of the natural monoterpene aldehyde safranal (1) and its reduction product safranol (4) were studied and the endoperoxide 5 from safranol was isolated and characterized by single crystal X-ray analysis. A dyade synthesis using DCC coupling of safranol with artesunic acid delivered the reactive substrate 7 for 1,3-diene photooxygenation. Singlet oxygen reaction of the substrate 7 enabled the synthesis of the trioxane-endoperoxide dyade 8. The diastereoisomeric 1,3-cyclohexadienes $\mathbf{1 0}$ were obtained from the proline-catalyzed Diels-Alder dimerization of the Michael aldehyde 9. From the singlet oxygen addition with the diastereoisomeric substrates 10, only the kinetically preferred cis-endoperoxide $\mathbf{1 1}$ was formed.
\end{abstract}

Keywords: Singlet oxygen, Diels-Alder reaction, cyclohexadienes, organocatalysis

\section{Introduction}

The singlet oxygen cycloaddition to conjugated cyclodienes is a highly controlled and reliable route to 1,4-oxyfunctionalized products. ${ }^{1}$ The primary reaction event is the formation of bicyclic 1,2-dioxenes (i.e. endoperoxides) by a concerted but asynchronous $4+2$ cycloaddition step. Ene reactions can sometimes compete with this cycloaddition route and for heterocyclic substrates such as thiophenes, pyrrols, oxazoles, and especially furans, the endoperoxide formation is exclusive. $^{2}$ There are also numerous naturally occurring 1,2-dioxenes, that are most probably not formed biosynthetically by singlet oxygen reactions, that exhibit interesting and diverse pharmacological properties. ${ }^{3}$ A well-known natural peroxide that can also be generated by singlet oxygen addition is ascaridole. ${ }^{4}$ This compound was used as anthelmintic drug and was among the first targets for the Schenck investigations on photooxygenations using sunlight 
irradiation of dye solutions which were very early examples of sustainable photocatalysis in synthesis. ${ }^{5}$

Our interest in this field of photochemistry arose from the search for new and effective antimalarial peroxides, an intensively investigated area in medicinal chemistry. ${ }^{6}$ The natural sesquiterpene artemisinin (qinhaosu), used for centuries in Chinese folk medicine as a plant extract, has initiated and stimulated this field of research. ${ }^{7}$ The enormous potency of the natural product that involves also anti-cancer drug activity just recently emerged. ${ }^{8,9}$ At the same time, however, reports appeared that describe the appearance of plasmodium species resistant against artemisinin and artemisinin-derivatives. ${ }^{10,11}$ Three approaches are currently under investigation to deal with this fatal trend: a) artemisinin combination therapy (ACT, with other non-peroxidic antimalarial drugs), ${ }^{12}$ b) new peroxidic substances following the structural prototype, ${ }^{13,14} \mathrm{c}$ ) dyade concepts ${ }^{15}$ involving structure combinations of two artemisinin monomers, ${ }^{16}$ artemisinin and quinolines, ${ }^{17}$ or artemisinin derivatives with synthetic $1,2,4$-trioxane structures. ${ }^{18,19}$ In this context, ascaridole was also described as one potential dyade partner molecule. ${ }^{20}$ To explore new structural motifs, we envisaged other natural occuring terpenoids with pre-endoperoxide structures. One attractive example is the trimethylated cyclohexadiene safranal 1. Safranal constitutes the major spice component of crocus flowers together with crocin, crocetin and picocrocetin, and is crucial for the aroma of saffron. ${ }^{21}$ We envisaged the singlet oxygen reaction with the aldehyde $\mathbf{1}$ as a route to an electrophilic building block for dyade coupling.

\section{Results and Discussion}

The photooxygenation of the aldehyde 1 in $\mathrm{CDCl}_{3}$ delivered after long reaction a mixture of the ene product 2 and the desired endoperoxide 3 (Scheme 1) together with unreacted starting material. Column chromatography allowed the isolation of $\mathbf{2}^{22}$ but unfortunately led to decomposition of the endoperoxide 3 . Therefore, we reversed the dyade concept and allocated the nucleophilic part to the endoperoxide component, i.e. conversion of the aldehyde safranal to the corresponding alcohol. ${ }^{23}$

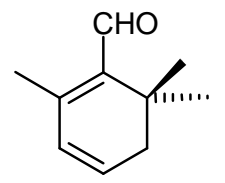

1

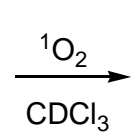

$\mathrm{CDCl}_{3}$

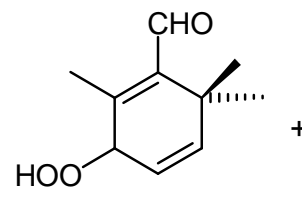

2

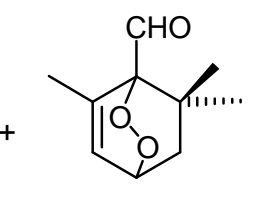

3

Scheme 1. Photooxygenation of natural safranal (1).

Near quantitative reduction of safranal (1) was achieved with $\mathrm{LiAlH}_{4}$, and the subsequent photooxygenation of the alcohol $\mathbf{4}$ in $\mathrm{CDCl}_{3}$ resulted in the endoperoxide $\mathbf{5}$ in a chemoselective singlet oxygen $4+2$ cycloaddition (Scheme 2 ). No ene products were detected in the crude NMR 
of the reaction mixture indicating that the hydroxymethyl group strongly activates the diene system towards $4+2$ cycloaddition.

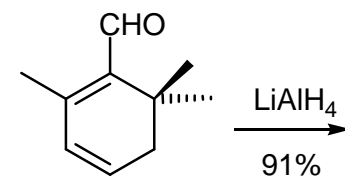

1<smiles>CC1=C(CO)[C@](C)(CO)CC=C1</smiles>

4<smiles>CC1=CC2C[C@](C)(COO2)C1(C)CO</smiles>

5

Scheme 2. Synthesis and photooxygenation of safranol (4).

The endoperoxide $\mathbf{5}$ was crystallized in racemic form from acetone and analyzed by crystal structure analysis (Figure 1). ${ }^{24}$ In contrast to the substrate 4 that is described as unstable, the endoperoxide $\mathbf{5}$ is completely stable at room temperature.

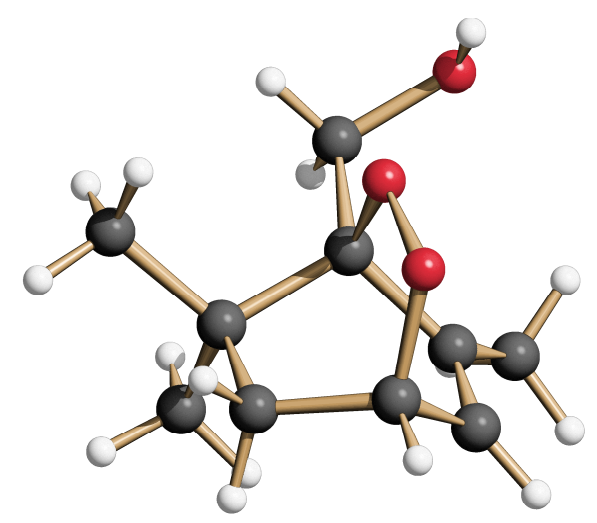

Figure 1. Structure of the endoperoxide 5 in the crystal. $^{23}$

To accomplish the dyade synthesis from endoperoxide $\mathbf{5}$ and an artemisinin skeleton, the Steglich esterification of $\mathbf{5}$ with artesunate (6) was investigated; a well-known process with numerous alcohols. ${ }^{25}$ In contrast to the literature reports coupling was not successful and led to Broensted-acid catalyzed decomposition of the alcohol component. Consequently, safranol was used as the alcohol component and esterified with artesunate using DCC/DMAP to give the ester 7 in 19\% isolated yield. Photooxygenation under standard conditions led to the desired artesunate-endoperoxide couple in $21 \%$ yield (Scheme 3 ). 


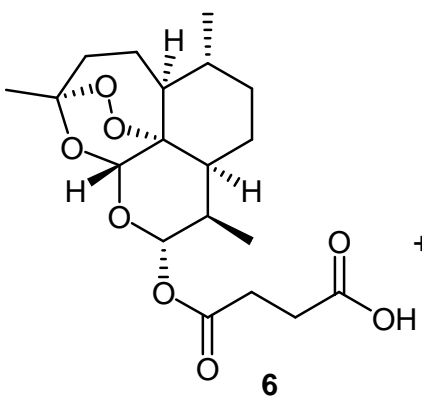<smiles>CC1[C@H](OC(=O)CCC(=O)O)O[C@H]2O[C@]3(C)CC[C@@H]1[C@H]2CC[C@H]3C</smiles>

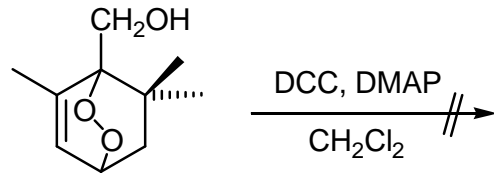

5
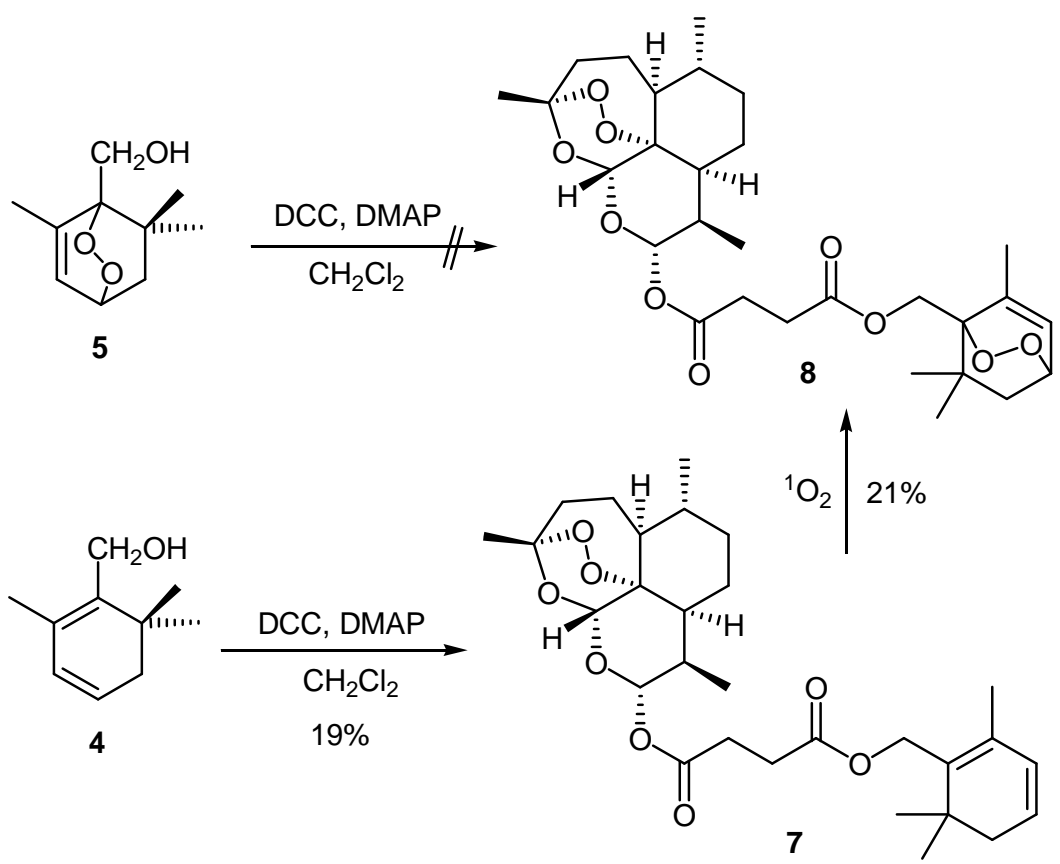

Scheme 3. Synthesis and photooxygenation of the artesunate-safranol dyade 7.

In the NMR spectra of the dyade $\mathbf{8}$ (Figure 2), the characteristic signals for the artemisinine skeleton - acetal H-11 and epimeric center H-12 - clearly show that 8 exists in an epimerically pure form with the ester substituent at $\mathrm{C}-12$ in anti-position to the methyl group at $\mathrm{C}-13$. The safranol endoperoxide part that is linked by the succinate chain appears as one set of signals in the ${ }^{1} \mathrm{H}$ as well as ${ }^{13} \mathrm{C}$ NMR (significant for carbon: the methylene group $\mathrm{C}-20$ and the stereogenic carbon center C-21). No single signal doubling was observed neither in the ${ }^{13} \mathrm{C}$ nor in the ${ }^{1} \mathrm{H}$ NMR spectra indicating that only one diastereoisomer of the dyade $\mathbf{8}$ was formed in the final photooxygenation process $(\mathbf{7} \rightarrow \mathbf{8})$.

During this study, we discovered an alternative approach to 1,3-cyclohexadiene-1carbaldehydes as substrates for dyade coupling. We performed these experiments in the context of organocatalytic photooxygenation reactions following the $\alpha$-hydroxylation route developed by Cordova et al. from aliphatic ketones and aldehydes, respectively, with singlet oxygen and amino acids as chiral organocatalysts. ${ }^{26,27}$ When these conditions were applied to $\alpha, \beta$-unsaturated aldehydes, complex mixtures of peroxides were observed that could not be separated. We expected the formation of dieneamines, as described in the work by Jørgensen et al. ${ }^{28,29}$ Obviously, we were not able to trap these dienes with singlet oxygen. Thus, the reaction conditions were modified and the photooxygenation was designed as a delayed step. When the Michael aldehyde 9 was treated with proline in the absence of any (additional) dienophile, the 1,3-cyclohexadiene carbaldehyde $\mathbf{1 0}$ was isolated as a mixture of trans/cis-isomers (3:2, enantioselectivity was not determined) in good yields. The rationale for this reaction is shown in Scheme 4 following the sequence that was described for intramolecular cycloaddition by Christmann and coworkers. ${ }^{30}$ During the subsequent reaction with singlet oxygen, one new set of 
signals appeared in the NMR spectra with concurrent disappearance of the signals of the cisisomer. After completion of the reaction, a complete conversion of cis-10 was observed with less than 5\% of the trans-endoperoxide $\mathbf{1 1}$ observable. The endoperoxide 11 with cis configuration, that could not be isolated from the reaction mixture, appeared in the ${ }^{13} \mathrm{C} N M R$ with a new indicative set of signals at 73.2 (C-4), 98.6 (C-1), 127.1 (C-5), and $135.1 \mathrm{ppm}$ (C-6). We are currently investigating this new synthetic approach to 1,3 -cyclohexadienes ${ }^{31}$ together with the unusual high stereoselective singlet oxygen cycloaddition.
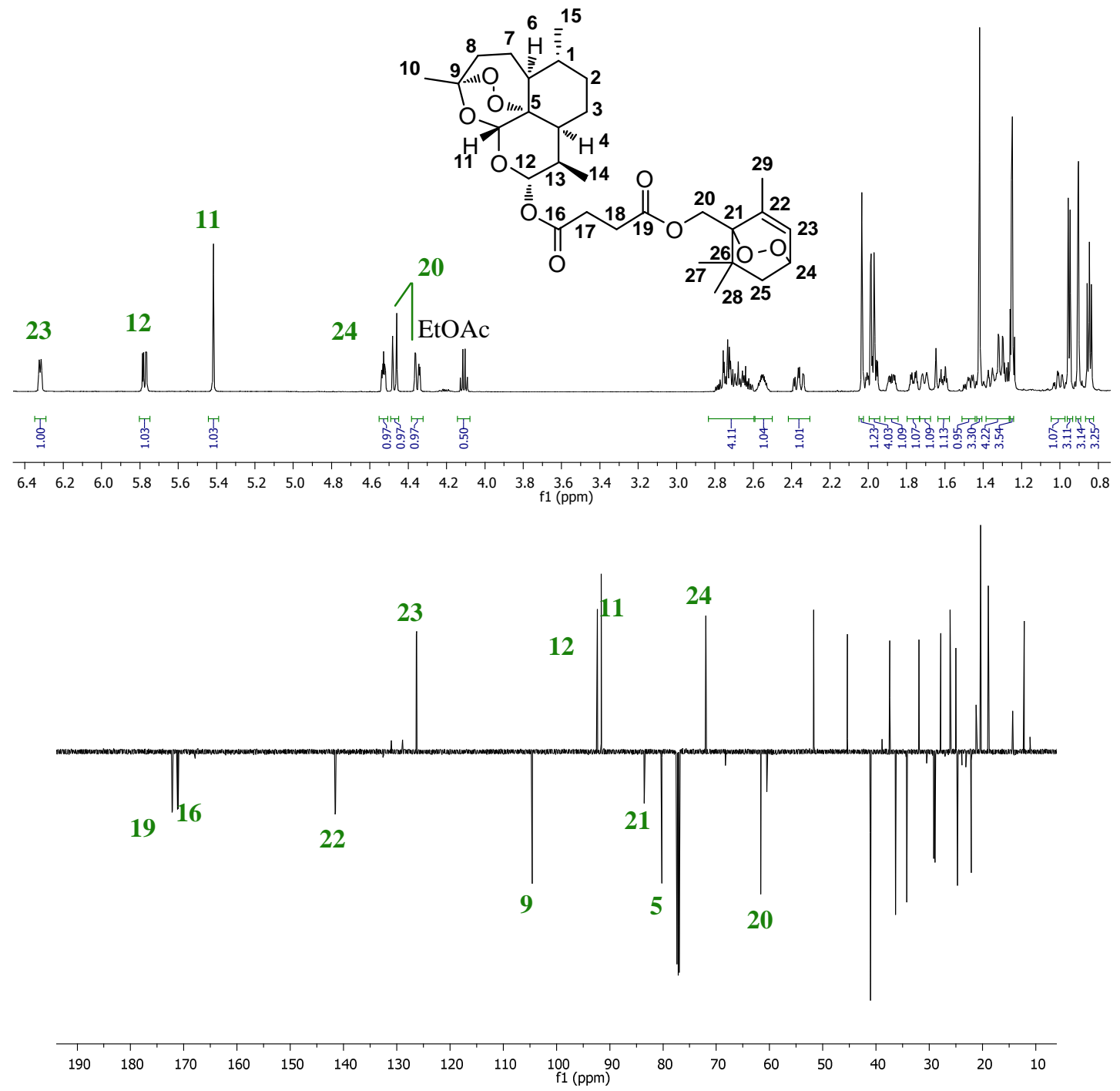

Figure 2. ${ }^{1} \mathrm{H}$ - and ${ }^{13} \mathrm{C}-\mathrm{NMR}$ of the trioxane-dioxane-dyade 8 . 


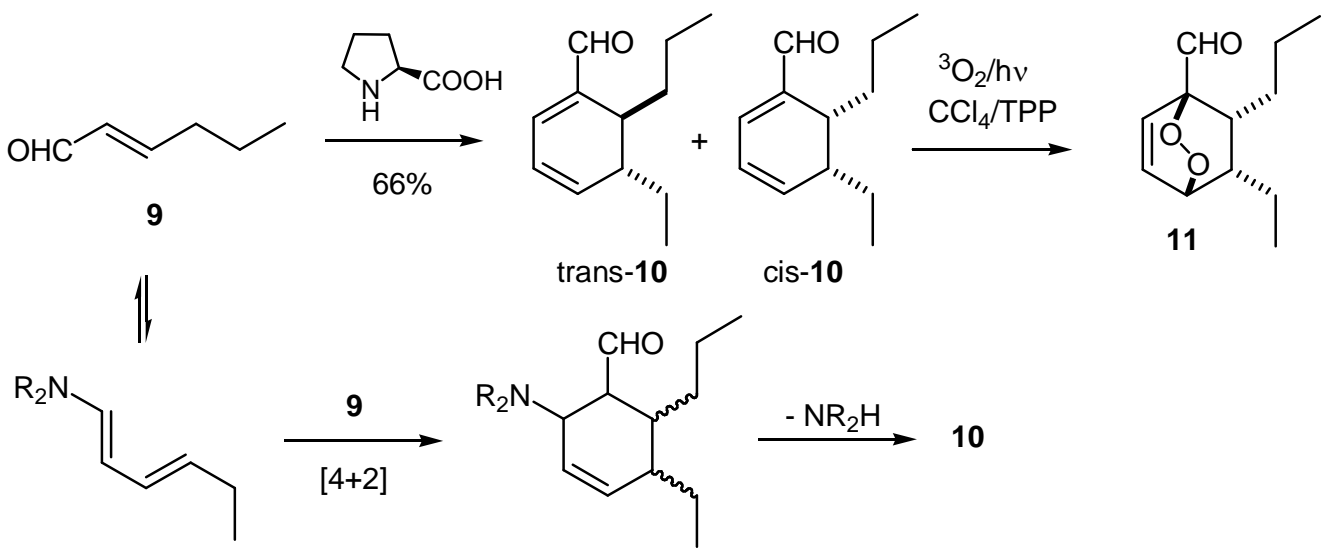

Scheme 4. Organocatalytic cycloaddition/elimination synthesis and diastereoselective photooxygenation of cyclohexadienes $\mathbf{1 0}$.

\section{Experimental Section}

General. Meso-tetraphenylporphyrin (TPP) was purchased from Porphyrin Systems, Bremen. The solvents for solution photooxygenation were puriss. and used as purchased. NMR spectra were recorded on Bruker DPX 300 and DPX 600 spectrometers, chemical shifts are given in $\delta$ (ppm) versus 0.0 (TMS for $\left.{ }^{1} \mathrm{H}\right)$ and $77.0\left(\mathrm{CDCl}_{3}\right.$ for $\left.{ }^{13} \mathrm{C}\right)$, multiplicities were determined by DEPT; IR spectra were obtained from a Perkin-Elmer 1600 series FTIR spectrometer; melting points were determined with a Büchi melting point apparatus (type Nr. 535) and are uncorrected; $\mathrm{CHN}$-combustion analyses were measured using an Elementar Vario EL instrument.

1-Hydroxymethyl-2,6,6-trimethylcyclohexa-1,3-diene (safranol, 4). To a suspension of 320 $\mathrm{mg}$ (8.32 mmol, 2.5 equiv.) of lithium aluminium hydride in dry diethyl ether (30 mL) externally cooled $0{ }^{\circ} \mathrm{C}$ was slowly added a solution of safranal $1(500 \mathrm{mg}, 3.33 \mathrm{mmol})$ in of diethyl ether $\left(20 \mathrm{~mL}\right.$ ). After stirring for $1 \mathrm{~h}$ at $0{ }^{\circ} \mathrm{C}$ and $1 \mathrm{~h}$ at room temperature, the reaction was quenched carefully with water and filtrated, extracted with diethyl ether $(3 \times 20 \mathrm{~mL})$ and dried $\left(\mathrm{MgSO}_{4}\right)$. After filtration and evaporation, safranol (4) (460 mg, 91\%) was obtained as colorless oil that was directly used for further reactions. ${ }^{1} \mathrm{H}$ NMR $\left(300 \mathrm{MHz}, \mathrm{CDCl}_{3}\right): 1.05\left(\mathrm{~s}, 6 \mathrm{H}, \mathrm{C}\left(\mathrm{CH}_{3}\right)_{2}\right), 1.81$ (s, 3H, $\left.\underline{\mathrm{C}}_{3}\right), 2.06$ (d, 2H, J $\left.2.6 \mathrm{~Hz}, 5-\mathrm{H}\right), 4.21\left(\mathrm{~s}, 2 \mathrm{H}, \mathrm{C}_{2} \mathrm{OH}\right), 5.69-5.79(\mathrm{~m}, 2 \mathrm{H}, 3-\mathrm{H}, 4-\mathrm{H}) ;{ }^{13} \mathrm{C}$ NMR (75 MHz, $\left.\mathrm{CDCl}_{3}\right)$ : 18.0 (q, $\left.\underline{\mathrm{CH}}_{3}\right), 26.7$ (q, $\left.\mathrm{C}\left(\underline{\mathrm{CH}}_{3}\right)_{2}\right), 33.4$ (s, C-6), 40.1 (t, C-5), 58.7 (t, $\left.\underline{\mathrm{CH}_{2}} \mathrm{OH}\right), 126.3(\mathrm{~d}, \mathrm{H} \underline{\mathrm{C}}=\mathrm{CH}), 129.1(\mathrm{~d}, \mathrm{HC}=\underline{\mathrm{CH}}), 129.3(\mathrm{~s}, \underline{\mathrm{C}}=\mathrm{C}), 137.4(\mathrm{~s}, \mathrm{C}=\underline{\mathrm{C}})$.

1-Hydroxymethyl-6,7,7-trimethyl-2,3-dioxabicyclo[2.2.2]oct-5-ene (5). A solution of safranol $4(228 \mathrm{mg}, 1.5 \mathrm{mmol})$ in $\left(2 \times 10^{-4} \mathrm{M}\right.$ in TPP $)$ deuterochloroform $(20 \mathrm{~mL})$ was irradiated at room temperature by means of a halogen metal vapour lamp under purging with dry oxygen and under TLC and NMR control for $8 \mathrm{~h}$. After evaporation of the solvent and column chromatography (silica, EtOAc/cyclohexane 1:4), the title compound $\mathbf{5}$ (150 mg, 54\%) was obtained as a colorless 
solid, mp 101-102 ${ }^{\circ} \mathrm{C} .{ }^{1} \mathrm{H}$ NMR (300 MHz, $\left.\mathrm{CDCl}_{3}\right): 0.76$ (s, 3H, - $\left.\left(\mathrm{C}_{3}\right)_{2}\right), 1.09$ (s, 3H, $\left.-\left(\mathrm{CH}_{3}\right)_{2}\right)$, 1.19 (dd, 1H, $J$ 13.1, $2.2 \mathrm{~Hz}, 8-\mathrm{H}), 1.81$ (dd, 1H, J 13.1, $\left.3.5 \mathrm{~Hz}, 8^{\prime}-\mathrm{H}\right), 1.99$ (d, 3H, J 1.6 Hz, $\left.\mathrm{C}_{3}\right), 3.78-3.89\left(\mathrm{~m}, 2 \mathrm{H}, \mathrm{CH}_{2} \mathrm{OH}\right), 4.38-4.43(\mathrm{~m}, 1 \mathrm{H}, 4-\mathrm{H}), 6.18(\mathrm{dd}, 1 \mathrm{H}, J 5.9,1.0 \mathrm{~Hz}, 5-\mathrm{H}) ;{ }^{13} \mathrm{C}$ NMR (75 MHz, $\left.\mathrm{CDCl}_{3}\right)$ : 18.6 (q, $\left.\underline{\mathrm{CH}}_{3}\right), 24.7$ (q, - $\left.\left(\underline{\mathrm{CH}}_{3}\right)_{2}\right), 27.3$ (q, - $\left.\left(\underline{\mathrm{C}} \mathrm{H}_{3}\right)_{2}\right), 33.6$ (s, C-7), 41.0 (t, C-8), 59.2 (t, $\underline{\mathrm{CH}}_{2} \mathrm{OH}$ ), 71.9 (d, C-4), 85.3 (s, C-1), 125.7 (d, C-5), 142.3 (s, C-6); HRMS (ESI, M+Na) Found: 207.0986, $\mathrm{C}_{10} \mathrm{H}_{16} \mathrm{O}_{3}$ requires 207.0992.

Dihydroartemisinyl(2,6,6-trimethylcyclohexa-1,3-dien-1-yl)methylsuccinate (7). A solution of artesunate $(7.69 \mathrm{~g}, 20.0 \mathrm{mmol})$ and DCC (4.33 g $21.0 \mathrm{mmol})$ in methylene chloride $(100 \mathrm{~mL})$ was cooled to $0{ }^{\circ} \mathrm{C}$. Over a period of $30 \mathrm{~min}$, a solution of safranol (4) $(3.04 \mathrm{~g}, 20 \mathrm{mmol})$ and DMAP (320 mg, $2.6 \mathrm{mmol}$ ) were added. The mixture was stirred for $12 \mathrm{~h}$ at room temp., then treated with a small amount of water and the separated organic was washed several times with water, separated and dried $\left(\mathrm{MgSO}_{4}\right)$. After evaporation of the solvent and column chromatography (silica, EtOAc/cyclohexane 1:8), the title compound 7 (1.94 g, 19\%) was obtained as a colorless oil. ${ }^{1} \mathrm{H}$ NMR $\left(600 \mathrm{MHz}, \mathrm{CDCl}_{3}\right): 0.82(\mathrm{~d}, 3 \mathrm{H}, J 7.2 \mathrm{~Hz}, 14-\mathrm{H}), 0.94$ (d, $3 \mathrm{H}, J 6.2 \mathrm{~Hz}, 15-\mathrm{H}), 0.97-1.03(\mathrm{~m}, 1 \mathrm{H}, 2-\mathrm{H}), 1.00(\mathrm{~s}, 6 \mathrm{H}, 27-\mathrm{H}, 28-\mathrm{H}), 1.80$ (s, 3H, 29-H), 1.23-1.49 (m, 4H, 6-H, 1-H, 3-H, 7-H), 1.41 (s, 3H, 10-H), 1.58-1.62 (m, 1H, 4-H), 1.67-1.72 (m, $1 \mathrm{H}, 2-\mathrm{H}), 1.73-1.78(\mathrm{~m}, 1 \mathrm{H}, 3-\mathrm{H}), 1.84-1.90(\mathrm{~m}, 1 \mathrm{H}, 7-\mathrm{H}), 1.98-2.04(\mathrm{~m}, 1 \mathrm{H}, 8-\mathrm{H}), 2.07$ (d, 2H, 25-H), 2.35 (dt, 1H, J 14.4, $3.9 \mathrm{~Hz}, 8-\mathrm{H}), 2.51-2.57(\mathrm{~m}, 1 \mathrm{H}, 13-\mathrm{H}), 2.61-2.75(\mathrm{~m}, 4 \mathrm{H}, 17-\mathrm{H}$, 18-H), 4.72 (s, 2H, 20-H), $5.42(\mathrm{~s}, 1 \mathrm{H}, 11-\mathrm{H}), 5.77$ (d, 1H, J $9.9 \mathrm{~Hz}, 12-\mathrm{H}), 5.75-5.81(\mathrm{~m}, 3 \mathrm{H}$, 12-H, 23-H, 24-H); ${ }^{13} \mathrm{C}$ NMR (150 MHz, $\mathrm{CDCl}_{3}$ ): 12.1 (q, C-14), 18.2 (q, C-29), 20.3 (q, C-15), 22.1 (t, C-3), 24.7 (t, C-7), 26.0 (q, C-10), 26.3 (q, Me), 28.8 (t, - $\underline{\mathrm{CH}}_{2} \mathrm{COOR}$ ), 29.0 (t, - $\underline{\mathrm{CH}}_{2} \mathrm{COOR}$ ), 29.1 (q, Me), 31.9 (d, C-13), 33.1 (s, C-26), 34.2 (t, C-2), 36.3 (t, C-8), 37.3 (d, C-1), 39.8 (t, C-25), 45.3 (d, C-4), 51.6 (d, C-6), 61.5 (t, C-20), 80.2 (s, C-5), 91.6 (d, C-11), 92.4 (d, C-12), 104.6 (s, C-9), 127.0 (d, HC= $=\mathrm{CH}$ ), 128.9 (d, $\mathrm{HC}=\mathrm{CH}), 131.8$ (s, $\mathrm{C}_{\mathrm{q}}$ ), 131.9 (s,

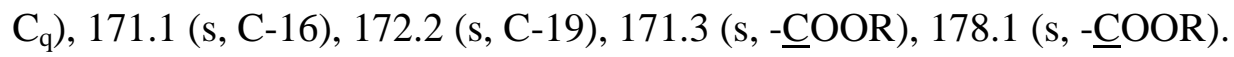

Dihydroartemisinyl[(6,7,7-trimethyl-2,3-dioxabicyclo[2.2.2] $]$ oct-5-en-1-yl)methyl]-succinate

(8). A solution of the dihydroartemisine $7(780 \mathrm{mg}, 1.5 \mathrm{mmol})$ in $\left(2 \times 10^{-4} \mathrm{M}\right.$ in TPP) of deuterochloroform $(20 \mathrm{~mL})$ and pyridine $(10 \mu \mathrm{L})$ was irradiated at room temperature by means of a halogen metal vapour lamp under purging with dry oxygen and under TLC and NMR control for $12 \mathrm{~h}$. Evaporation of the solvent and column chromatography (silica, EtOAc/cyclohexane 1:6) afforded the title compound $\mathbf{8}(170 \mathrm{mg}, 21 \%)$ as a colorless solid, mp 167-168 (decomp.); ${ }^{1} \mathrm{H}$ NMR (600 MHz, $\mathrm{CDCl}_{3}$ ): 0.82 (d, 3H, J 7.1 Hz, 14-H), 0.90 (d, 3H, J 1.9 $\mathrm{Hz}, 27-\mathrm{H}), 0.94$ (d, 3H, J 6.2 Hz, 15-H), 0.97-1.03 (m, 1H, 2-H), 1.23-1.49 (m, 4H, 6-H, 1-H, 3-H, 7-H), 1.24-1.25 (m, 3H, 28-H), 1.27-1.39 (m, 4H, 6-H, 25-H, 1-H, 3-H), 1.41 (s, 3H, 10-H), 1.58-1.62 (m, 1H, 4-H), 1.67-1.72 (m, 1H, 2-H), 1.73-1.78 (m, 1H, 3-H), 1.84-1.90 (m, 1H, 7-H), 1.95-2.00 (m, 4H, 25-H, 29-H), 1.98-2.04 (m, 1H, 8-H), 2.35 (dt, 1H, J 14.4, $3.9 \mathrm{~Hz}$, 8-H), 2.51-2.57 (m, 1H, 13-H), 2.61-2.75 (m, 4H, 17-H, 18-H), 4.35 (dd, 1H, J 12.6, $2.4 \mathrm{~Hz}$, 20-H), 4.52-4.54 (m, 1H, 24-H), $5.42(\mathrm{~s}, 1 \mathrm{H}, 11-\mathrm{H}), 5.77$ (d, 1H, J $9.9 \mathrm{~Hz}, 12-\mathrm{H}), 6.32(\mathrm{~d}, 1 \mathrm{H}, J$ $6.1 \mathrm{~Hz}, 23-\mathrm{H}) ;{ }^{13} \mathrm{C}$ NMR (150 MHz, $\mathrm{CDCl}_{3}$ ): 12.1 (q, C-14), 18.9 (q, C-29), 20.3 (q, C-15), 22.1 (t, C-3), 24.7 (t, C-7), 25.0 (q, C-28), 26.0 (q, C-10), 27.9 (q, C-27), 28.8 (t, - $\underline{\mathrm{CH}}_{2} \mathrm{COOR}$ ), 29.0 
(t, - $\underline{\mathrm{CH}}_{2} \mathrm{COOR}$ ), 31.9 (d, C-13), 34.2 (t, C-2), 34.3 (s, C-26), 36.3 (t, C-8), 37.3 (d, C-1), 41.1 (t, C-25), 45.3 (d, C-4), 51.6 (d, C-6), 61.1 (t, C-20), 72.0 (d, C-24), 80.2 (s, C-5), 83.6 (s, C-22), 91.6 (d, C-11), 92.4 (d, C-12), 104.6 (s, C-9), 126.3 (d, C-23), 140.6 (s, C-21), 171.1 (s, -

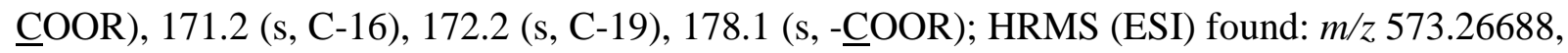
$\mathrm{C}_{29} \mathrm{H}_{40} \mathrm{O}_{10}$ requires 573.26701.

5-Ethyl-6-propylcyclohexa-1,3-dienecarbaldehyde (10). A solution of trans-2-hexenal (9) (1.0 $\mathrm{g}, 10 \mathrm{mmol})$ in methanol $(30 \mathrm{~mL})$ of was treated with $(S)$-proline $(115 \mathrm{mg}, 1.00 \mathrm{mmol})$ and stirred at room temp. for $24 \mathrm{~h}$. The solvent was subsequently evaporated and the residue extracted with EtOAc $(20 \mathrm{~mL})$, washed with water $(20 \mathrm{~mL})$ and extracted again with EtOAc $(2 \times$ $20 \mathrm{~mL}$ ). Column chromatography (cyclohexane/EtOAc 3:1, $R_{\mathrm{f}} 0.48$ ) gave a mixture of diastereoisomeric cyclohexadienes 10 (400 mg, 45\%, trans:cis 65:35) as a colorless oil. ${ }^{1} \mathrm{H}$ NMR (300 MHz, $\mathrm{CDCl}_{3}$, trans-10): 0.81-0.96 (m, 6H, CH-CH $\left.{ }_{2}-\mathrm{CH}_{3} / \mathrm{CH}_{2}-\mathrm{CH}_{2}-\mathrm{C}_{3}\right), 1.16-1.42$ (m, $\left.6 \mathrm{H}, \mathrm{CH}-\underline{\mathrm{C}}_{2}-\mathrm{CH}_{3} / \underline{\mathrm{C}}_{2}-\underline{\mathrm{CH}}_{2}-\mathrm{CH}_{3}\right), 2.18\left(\mathrm{q}, 1 \mathrm{H}, J 6.6 \mathrm{~Hz} \mathrm{C}-\mathrm{CH}_{2}-\mathrm{CH}_{3}\right), 2.69$ (t, $1 \mathrm{H}, J 6.6 \mathrm{~Hz}$ C $\underline{H}-\mathrm{C}-\mathrm{C}=\mathrm{O}), 6.11\left(\mathrm{dd}, 1 \mathrm{H}, J\right.$ 9.6, $\left.5.2 \mathrm{~Hz}, \mathrm{CH}-\mathrm{CH}_{\mathrm{sp} 2}-\mathrm{C}_{\mathrm{sp} 2}\right), 6.23$ (dd, $1 \mathrm{H}, J$ 9.3, $5.9 \mathrm{~Hz}, \mathrm{CH}-$ $\left.\underline{\mathrm{CH}}_{\mathrm{sp} 2}-\mathrm{CH}_{\mathrm{sp} 2}\right), 6.66\left(\mathrm{~d}, 1 \mathrm{H}, J 5.2 \mathrm{~Hz}, \mathrm{C}_{\mathrm{sp} 2}-\mathrm{C}-\mathrm{C}=\mathrm{O}\right), 9.46(\mathrm{~s}, 1 \mathrm{H}, \mathrm{CHO}) ;{ }^{13} \mathrm{C} \mathrm{NMR}(75 \mathrm{MHz}$, $\mathrm{CDCl}_{3}$, trans-10): 11.2 (q, 1C, $\mathrm{CH}-\mathrm{CH}_{2}-\underline{\mathrm{CH}}_{3}$ ), 14.2 (q, $1 \mathrm{C}, \mathrm{CH}-\mathrm{CH}_{2}-\mathrm{CH}_{2}-\underline{\mathrm{CH}_{3}}$ ), 19.9 (t, $1 \mathrm{C}, \mathrm{CH}-$

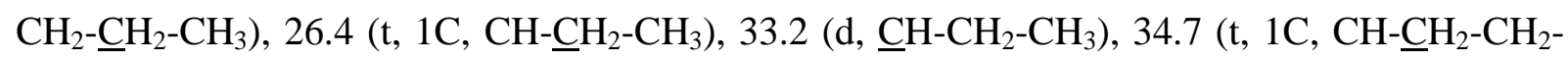
$\mathrm{CH}_{3}$ ), 39.7 (d, $\left.1 \mathrm{C}, \underline{\mathrm{C}} \mathrm{H}-\mathrm{CH}_{2}-\mathrm{CH}_{2}-\mathrm{CH}_{3}\right), 122.0$ (d, 1C, $\left.\mathrm{CH}_{\mathrm{sp} 3}-\mathrm{CH}-\underline{\mathrm{C}} \mathrm{H}\right), 141.0$ (d, 1C, $\mathrm{CH}_{\mathrm{sp} 3}-\underline{\mathrm{C}} \mathrm{H}$ ), 141.2 (s, 1C, $\underline{\mathrm{C}}-\mathrm{CHO}$ ), 141.3 (d, 1C, CH-C-CHO), 193.5 (d, 1C, CHO); ${ }^{1} \mathrm{H}$ NMR (300 MHz, $\mathrm{CDCl}_{3}$, cis-10): 0.81-0.96 (m, 6H, CH-CH $\left.{ }_{2}-\underline{\mathrm{C}}_{3} / \mathrm{CH}_{2}-\mathrm{CH}_{2}-\mathrm{C}_{3}\right), 1.16-1.42$ (m, 6H, CH-C $\underline{H}_{2}-$ $\mathrm{CH}_{3} / \mathrm{CH}_{2}-\underline{\mathrm{C}}_{2}-\mathrm{CH}_{3}$ ), 2.33-2.46 (m, 1H, $\underline{\mathrm{C}}-\mathrm{CH}_{2}-\mathrm{CH}_{3}$ ), 2.74-2.84 (m, C$\left.-\mathrm{C}-\mathrm{CHO}\right), 6.00$ (d, $1 \mathrm{H}$, $\left.J 9.5 \mathrm{~Hz}, \mathrm{CH}-\mathrm{CH}_{\mathrm{sp} 2}-\underline{\mathrm{CH}}_{\mathrm{sp} 2}\right), 6.10-6.20\left(\mathrm{~m}, 1 \mathrm{H}, J\right.$ 9.3, $\left.5.9 \mathrm{~Hz}, \mathrm{CH}-\underline{\mathrm{CH}}_{\mathrm{sp} 2}-\mathrm{CH}_{\mathrm{sp} 2}\right), 6.70$ (d, 1H, J 5.1 $\left.\mathrm{Hz}, \mathrm{C}_{\mathrm{sp} 2}-\mathrm{C}-\mathrm{C}=\mathrm{O}\right), 9.52$ (s, 1H, CHO); ${ }^{13} \mathrm{C} \mathrm{NMR}\left(75 \mathrm{MHz}, \mathrm{CDCl}_{3}\right.$, cis-10): 12.0 (q, 1C, CH$\mathrm{CH}_{2}-\mathrm{CH}_{3}$ ), 14.8 (q, 1C, CH- $\mathrm{CH}_{2}-\mathrm{CH}_{2}-\underline{\mathrm{CH}}_{3}$ ), 20.7 (t, 1C, CH-CH $-\mathrm{CH}_{2}-\mathrm{CH}_{3}$ ), 23.3 (t, 1C, CH-

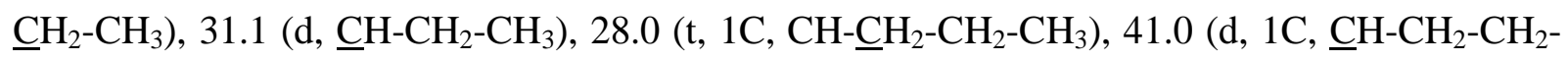
$\mathrm{CH}_{3}$ ), 123.8 (d, 1C, $\mathrm{CH}_{\mathrm{sp} 3}-\mathrm{CH}-\underline{\mathrm{CH}}$ ), 142.1 (d, 1C, $\mathrm{CH}_{\mathrm{sp} 3}-\underline{\mathrm{CH}}$ ), 141.1 (d, 1C, C-CHO), 143.7 (s, 1C, C-CHO), $193.2(\mathrm{~d}, 1 \mathrm{C}, \mathrm{CHO}) . \mathrm{GC}-\mathrm{MS}: \tau_{\mathrm{R}}\left(\right.$ trans-10) $=9.82 \mathrm{~min} ; \mathrm{m} / z(\%) 178\left(35 ;[\mathrm{M}]^{+}\right)$, 149 (13), 145 (10), 135 (12), 119 (10), 107 (40), 91 (31), 79 (100), 65 (9), 51 (7); $\tau_{\mathrm{R}}($ cis-10) = $10.16 \mathrm{~min} ; \mathrm{m} / \mathrm{z}(\%) 178\left(21 ;[\mathrm{M}]^{+}\right), 149$ (9), 141 (12), 133 (13), 119 (11), 107 (30), 94 (42), 79 (100), 65 (25), 55 (19).

\section{Acknowledgements}

This research was supported by the Deutsche Forschungsgemeinschaft and by University start-up funding. 


\section{References}

1. Iesce, M. R.; Cermola, F. CRC Handbook of Organic Photochemistry and Photobiology, 3rd edition (Griesbeck, A. G.; Oelgemoller, M.; Ghetti, F., eds.) CRC Press, 2012, 1, 727.

2. Montagnon, T.; Kalaitzakis, D.; Triantafyllakis, M.; Stratakis, M.; Vassilikogiannakis, G. Chem. Commun. 2014, 50, 15480. http://dx.doi.org/10.1039/C4CC02083A

3. O'Neill , P. M.; Posner, G. H. J. Med. Chem. 2004, 47, 2945. http://dx.doi.org/10.1021/jm030571c

4. Johnson, M. A.; Croteau, R. Arch. Biochem. Biophys. 1984, 235, 254. http://dx.doi.org/10.1016/0003-9861(84)90274-1

5. Schenck, G. O.; Kinkel, K. G.; Mertens, H. J. Justus Liebigs Ann. Chem. 1953, 584, 125. http://dx.doi.org/10.1002/jlac.19535840110

6. Slack, R. D.; Jacobine, A. M.; Posner, G. H. Med. Chem. Commun. 2012, 3, 281. http://dx.doi.org/10.1039/c2md00277a

7. Haynes, R. K. Curr. Opin. Infect. Dis. 2001, 14, 719. http://dx.doi.org/10.1097/00001432-200112000-00010

8. Chaturvedi, D.; Goswami, A.; Saikia, P. P.; Barua, N. C.; Rao, P. G. Chem. Soc. Rev. 2010, 39, 435. http://dx.doi.org/10.1039/b816679j

9. Also following the hybrid molecule approach: Reiter, C.; Karagöz, A. C.; Fröhlich, T.; Klein, V.; Zeino, M.; Viertel, K.; Held, J.; Mordmüller, B.; Öztürk, S. E.; Anil, H.; Efferth, T.; Tsogoeva, S. B. Eur. J. Med. Chem. 2014, 75, 403. http://dx.doi.org/10.1016/j.ejmech.2014.01.043

10. Sá, J. M.; Chong , J. L.; Wellems, T. E. Essays Biochem. 2011, 51, 137.

11. Meshnick, S. R. Int. J. Parasitol. 2002, 32, 1655. http://dx.doi.org/10.1016/S0020-7519(02)00194-7

12. Cui, L.; Su, X.-Z. Expert Rev. Anti-Infect. Ther. 2009, 7, 999. http://dx.doi.org/10.1586/eri.09.68

13. Singh, C.; Pandey, S.; Sharma, M.; Puri, S. K. Bioorg. Med. Chem. 2008, 16, 1816. http://dx.doi.org/10.1016/j.bmc.2007.11.012

14. Kumar, N.; Sharma , M.; Rawat, D. S. Curr. Med. Chem. 2011, 18, 3889. http://dx.doi.org/10.2174/092986711803414340

15. Meunier, B. Acc. Chem. Res. 2008, 41, 69. http://dx.doi.org/10.1021/ar7000843

16. Posner, G. H.; Paik, I. H.; Chang, W.; Bortnik, K.; Sinishtaj, S.; Rosenthal , A. S.; Shapiro, T. A. J. Med. Chem. 2007, 50, 2516. http://dx.doi.org/10.1021/jm070149m

17. Dechy-Cabaret, O.; Benoit-Vical, F.; Robert, A.; Meunier, B. ChemBioChem 2000, 1, 281. http://dx.doi.org/10.1002/1439-7633(20001117)1:4<281::AID-CBIC281>3.0.CO;2-W 
18. Griesbeck, A. G.; Neudörfl, J.; Hörauf, A.; Specht, S.; Raabe, A. J. Med. Chem. 2009, 52, 3420 . http://dx.doi.org/10.1021/jm9002523

19. Griesbeck, A. G.; Raabe, A. Synlett 2009, 1514. http://dx.doi.org/10.1055/s-0029-1216740

20. Hatzakis, E.; Opsenica, I.; Solaja, B. A.; Stratakis, M. Arkivoc 2007, (viii), 124.

21. Cullere, L.; San-Juan, F.; Cacho, J. Food Chem. 2011, 127, 1866. http://dx.doi.org/10.1016/j.foodchem.2011.02.015

22. Characterized as the corresponding hydroxyaldehyde reduction product: Brémond, P.; Audran, G.; Juspin, T.; Monti, H. Eur. J. Org. Chem. 2007, 2802. http://dx.doi.org/10.1002/ejoc.200601080

23. Karvinen, E. K.; Koskinen, A. M. P. Tetrahedron 1995, 51, 7555. http://dx.doi.org/10.1016/0040-4020(95)00378-L

24. X-ray structure of 5 (Cambridge Crystallographic Data Centre deposition number CCDC 1030726): empirical formula $\mathrm{C} 10 \mathrm{H} 16 \mathrm{O} 3$, formula weight: 184.23 , space group tetragonal: P41, unit cell dimensions: $\mathrm{a}=12.2264(6) \AA, \alpha=\beta=\gamma=90^{\circ}, \mathrm{b}=12.2264(6) \AA, \mathrm{c}=6$. 7060(2) $\AA, \mathrm{Z}=4, \mathrm{~V}=1002.45 \AA 3$, final $\mathrm{R}$ indices $[\mathrm{I}>2 \square(\mathrm{I})] \mathrm{R} 1=0.0352$, wR2 = 0.0834; $\mathrm{R}$ indices (all data) $\mathrm{R} 1=0.0512, \mathrm{wR} 2=0.1027$, number of reflexes: 6268, independent reflexes: 1185 , reflexes with $\mathrm{I}>2 \sigma(\mathrm{I}): 1039$.

25. Haynes, R. K.; Chan, H.-W.; Cheung, M.-K.; Lam, W.-L.; Soo, M.-K.; Tsang, H.-W.; Voerste, A.; Williams, I. D. Eur. J. Org. Chem. 2002, 113. http://dx.doi.org/10.1002/1099-0690(20021)2002:1<113::AID-EJOC113>3.0.CO;2-N

26. Sunden, H.; Engqvist, M.; Casas, J.; Ibrahem, I.; Cordova, A. Angew. Chem., Int. Ed. 2004, 43,6532 . http://dx.doi.org/10.1002/anie.200460295

27. Cordova, A.; Sunden, H.; Engqvist, M.; Ibrahem, I.; Casas, J. J. Am. Chem. Soc. 2004, 126, 8914. http://dx.doi.org/10.1021/ja047930t

28. Bertelsen, S.; Marigo, M.; Brandes, S.; Diner, P.; Jørgenson, K. A. J. Am. Chem. Soc. 2006, $128,12973$. http://dx.doi.org/10.1021/ja064637f

29. Brandau, S.; Landa, A.; Franzen, J.; Marigo, M.; Jørgenson, K. A. Angew. Chem., Int. Ed. 2006, $45,4305$.

http://dx.doi.org/10.1002/anie.200601025

30. De Figueiredo, R. M.; Fröhlich, R.; Christmann, M. Angew. Chem., Int. Ed. 2008, 47, 1450. http://dx.doi.org/10.1002/anie.200704688

31. Analogous products are obtained only by multistep processes: Kündig, E. P.; Ripa, A.; Liu, R.; Bernardinelli, G. J. Org. Chem. 1994, 59, 4773.

http://dx.doi.org/10.1021/jo00096a019 